\title{
La hantise de la banqueroute : les finances publiques dans la période franco-batave (1795-1810)
}

\section{Tom Pfeil}

\author{
(2) OpenEdition \\ Journals \\ Édition électronique \\ URL : https://journals.openedition.org/ahrf/472 \\ DOI : 10.4000/ahrf.472 \\ ISSN : 1952-403X \\ Éditeur : \\ Armand Colin, Société des études robespierristes \\ Édition imprimée \\ Date de publication : 1 décembre 2001 \\ Pagination : 53-64 \\ ISSN : 0003-4436 \\ Référence électronique \\ Tom Pfeil, « La hantise de la banqueroute : les finances publiques dans la période franco-batave \\ (1795-1810) », Annales historiques de la Révolution française [En ligne], 326 | octobre- décembre 2001, \\ mis en ligne le 10 mars 2008, consulté le 23 avril 2022. URL : http://journals.openedition.org/ahrf/472 \\ ; DOI : https://doi.org/10.4000/ahrf.472
}




\title{
LA HANTISE DE LA BANQUEROUTE LES FINANCES PUBLIQUES DANS LA PÉRIODE FRANCO-BATAVE (1795-1810)
}

\author{
TOM PFEIL
}

\begin{abstract}
La République batave se distingue par son caractère pragmatique, en ce sens qu'elle n'est pas réellement dominée par l'idéologie mais par les finances. C'est qu'au cours du XVIIle siècle, les Pays-Bas étaient en crise ; ils perdirent leur crédit et une grande partie de leurs débouchés. II fallait donc pour survivre réformer le système financier, et, notamment la fiscalité. Cette mesure allait dans le sens de la révolution. À savoir dans le sens de la centralisation et de l'uniformisation.
\end{abstract}

Mots clés : République batave ; finances ; crise ; déficit ; fiscalité ; banqueroute.

Les Néerlandais n'ont jamais ressenti le désir de commémorer les années 1795 et 1798 (1). C'est pourtant la révolution de 1795 et le gouvernement d'État de 1798 qui posèrent les fondements de l'État unitaire néerlandais actuel. Ces deux années symbolisent la fin de l'Ancien Régime des Provinces-Unies et la naissance d'un nouvel ordre politique et administratif. Elles symbolisent également le caractère révolutionnaire de la période franco-batave (1795-1810), pendant laquelle une nouvelle génération politique de bourgeois lettrés, influencée par l'idéologie des Lumières, débattait publiquement sur la forme à donner au régime (républicain). Dans le processus de formation de l'État moderne des Pays-Bas, ces années sont incontournables. Non seulement en ce qui concerne la structure institutionnelle où allait se jouer la vie politique, mais aussi pour ce qui est de la culture politique, c'est-à-dire l'ensemble des conceptions, des discours et des pratiques qui confèrent un contenu et une forme à la vie politique.

(1) Cet article est pour une grande part inspiré de ma thèse, publiée sous le titre : Tot redding van het vaderland Het primaat van de Nederlandse overheidsfinanciën in de Bataafs-Franse tijd (1795-1810), Amsterdam, Neha, 1998. Pour plus de sources et de références, se référer à cet ouvrage. 
L'histoire politique des Pays-Bas à l'époque révolutionnaire de la fin du $\mathrm{XVIII}^{\mathrm{e}}$ et du début du XIX ${ }^{\mathrm{e}}$ siècle (1780-1815) n'est pourtant pas dominée par l'idéologie mais par les finances. Le problème des finances publiques et du financement des guerres a joué un rôle déterminant aussi bien dans la chute de la République des Provinces-Unies en 1795 que dans la construction de l'État unitaire néerlandais dans la période qui suit. L'effondrement de l'ancienne République découle en grande partie des problèmes structurels que rencontraient les finances publiques à la fin du XVIII ${ }^{\mathrm{e}}$ siècle ; problèmes qui aboutirent à la chute du crédit hollandais dans les années 1793-1795. Dans la période suivante, la reconstruction de l'État néerlandais fut par conséquent confrontée à la nécessité politique de restaurer et de renforcer le crédit public. Pour que survive ou subsiste la République batave, il était d'une importance vitale que les dépenses (croissantes) dues aux guerres et au paiement de la dette puissent être financées quelles que soient les circonstances. En ce sens, la nécessité de restaurer le crédit public eut un impact essentiel sur la modernisation (radicale) du système politique et financier des Pays-Bas.

\section{L'héritage politique et financier}

Les Bataves, qui vinrent au pouvoir après 1795, étaient traumatisés par les craintes et les incertitudes du siècle qui allait clore. Après s'être hissée durant le Siècle d'Or au rang de grande puissance européenne, au cours du XVIII $^{\mathrm{e}}$ siècle, la République des Provinces-Unies semblait devoir redescendre au niveau des petits États. L'issue de la guerre de Succession d'Espagne (1701-1713), celle de la guerre de Succession d'Autriche (1740-1748) et la défaite contre l'Angleterre (1780-1784) témoignaient que la confédération néerlandaise était de moins en moins capable de défendre ses intérêts politiques et économiques en Europe et ses colonies d'outre-mer par des moyens diplomatiques et militaires. Les problèmes financiers surtout encouragèrent les États Généraux de la République à adopter une politique de neutralité dans les conflits internationaux (Tableau 1).

La plupart des hommes politiques bataves pensaient que le déclin néerlandais était dû en majeure partie à l'affaiblissement du pouvoir des anciennes administrations provinciales, aux défauts des méthodes de financement utilisées par la Généralité et à la corruption en vigueur dans le gouvernement du stadhouder et des régents. La génération patriote de 1795 était donc en principe convaincue que le déclin politique de la République ne pouvait être freiné que par l'instauration d'une «démocratie représentative», afin que soient restaurée la légitimité politique ; constitué un État unitaire pour renforcer le pouvoir central ; introduits des impôts uniformes pour remédier au déficit des finances publiques. La réforme du système financier et fiscal surtout était vue 
Tableau 1. Vue d'ensemble des recettes, dépenses et dettes ordinaires et extraordinaires, en Hollande dans les années 1670,1721,1750,1790 et 1795 (en millions de florins).

(Pfeil, Ibid., p.49)

\begin{tabular}{|l|l|l|l|l|l|}
\hline année & $\mathbf{1 6 7 0}$ & $\mathbf{1 7 2 1}$ & $\mathbf{1 7 5 0}$ & $\mathbf{1 7 9 0}$ & $\mathbf{1 7 9 5}$ \\
\hline $\begin{array}{l}\text { Recettes } \\
\text { (ordinaires) }\end{array}$ & 11,2 & 12,9 & 13,3 & 15,8 & 14,9 \\
$\begin{array}{l}\text { Dépenses } \\
\text { (ordinaires) }\end{array}$ & 11,1 & 19,8 & 24,2 & 25,2 & 26,4 \\
Intérêts & 5,7 & 13,7 & 14,9 & 16,1 & $(17,1)$ \\
$\begin{array}{l}\text { Déficit } \\
\text { (ordinaire) }\end{array}$ & $-0,1$ & 6,9 & 10,9 & 9,4 & 11,5 \\
$\begin{array}{l}\text { Recettes } \\
\text { (extraordinaires) }\end{array}$ & - & 6,2 & 8,7 & 8,4 & 7,9 \\
$\begin{array}{l}\text { Dépenses } \\
\text { (extraoordinaires) }\end{array}$ & - & - & 0,6 & 7,4 & 3,6 \\
Total déficit & - & 0,7 & 2,8 & 8,3 & 7,2 \\
\hline
\end{tabular}

comme prioritaire. Mais à une époque où sévissait en Europe de façon permanente la guerre, il était plus que difficile de réformer la politique et les finances. La guerre n'était pas du reste le seul obstacle que devaient affronter les patriotes. Ce qui entravait la réussite de toute réforme, c'était aussi la dette publique (dettes et intérêts), les énormes contributions exigées par la France en numéraire et les divisions politiques internes. Le changement de pouvoir de 1795 promettait une reprise de confiance entre l'État et le capital ; confiance qui s'était détériorée depuis 1787. Éloquent à ce sujet est le fait que les Bataves reconnurent sans broncher les dettes de l'Ancien Régime. Mais du coup, la République batave se retrouva avec un héritage empoisonné : une dette dont les intérêts absorbaient plus de la moitié des recettes annuelles (Tableau 2).

Tableau 2. Dette, intérêts et recettes fiscales dans la République batave vers 1795, en millions de florins, suivis d'une subdivision par province en millions de florins et par tête (provinciale) en florins. (Pfeil, Ibid., p. 122).

\begin{tabular}{|l|r|r|c|c|c|}
\hline & dette & intérêts & $\begin{array}{c}\text { recettes } \\
\text { fiscales }\end{array}$ & $\begin{array}{c}\text { intérêts } \\
\text { par tête }\end{array}$ & $\begin{array}{c}\text { recettes } \\
\text { fiscales par tête }\end{array}$ \\
\hline Hollande & 454,0 & 12,50 & 18,14 & 15,70 & 22,84 \\
Gelderland & 8,8 & 0,35 & 1,59 & 1,56 & 7,14 \\
Overijssel & 9,4 & 0,33 & 0,93 & 2,43 & 6,88 \\
Groningue & 9,7 & 0,35 & $\mathbf{1 , 3 9}$ & 3,52 & 12,18 \\
Zélande & 58,3 & 1,62 & 2,09 & 14,10 & 18,25 \\
Frise & 31,7 & 1,23 & 3,03 & $\mathbf{7 , 6 2}$ & 18,77 \\
Utrecht & 37,3 & 0,86 & $\mathbf{1 , 7 9}$ & 8,83 & 18,51 \\
Drenthe & 2,0 & 0,07 & 0,20 & 1,76 & 5,04 \\
Brabant hollandais & $\mathbf{1 , 8}$ & 0,06 & $\mathbf{1 , 2 5}$ & 0,23 & 4,80 \\
Généralité & $\mathbf{1 7 , 5}$ & 0,70 & 2,40 & & \\
CIO & 120,9 & 4,08 & & & \\
Amirautés & $\mathbf{1 6 , 0}$ & 0,80 & & & \\
Total & $\mathbf{7 6 7 , 4}$ & $\mathbf{2 2 , 9 5}$ & $\mathbf{3 2 , 8 1}$ & $\mathbf{1 1 , 8 1}$ & $\mathbf{1 5 , 6 7}$ \\
\hline
\end{tabular}


Les données du tableau 2 montrent bien que la structure financière et fiscale de la République était extrêmement hétérogène, étant donné les différences provinciales en matière de dettes, de recettes et d'arrérages d'intérêts. Ces différences grevaient la réalisation d'un système uniforme de fiscalité et de financement. Les intérêts financiers des diverses provinces étaient diamétralement opposés. Aussi les provinces qui contribuaient le plus à supporter les charges communes pouvaient-elles se réjouir d'une unification financière de la République batave, tandis qu'en principe les autres en feraient les frais. La voie vers l'unification financière était pavée d'autres obstacles encore. Le premier, et non le moindre, on l'a entrevu, était celui qu'imposait l'alliance française (2). Le premier défi auquel fut confronté le nouveau gouvernement batave fut d'endiguer le flot d'assignats dépréciés, qui, depuis l'entrée des troupes françaises en Hollande, inondaient le pays. Pour son aide dans la «libération» du pays, le gouvernement français exigea également une rétribution en argent d'une somme d'un million de florins. Cette contribution de guerre ou idemnité particulièrement lourde, qui équivalait aux recettes de trois années d'impôts, fut stipulée dans le Traité de La Haye, conclu le 16 mai 1795 entre la France et la Hollande, après bien des négociations laborieuses (3). La ratification du traité de paix de la Haye impliquait également que la République batave contractait une alliance offensive et défensive avec la France. En liant ainsi son sort à celui de la France, l'État batave s'engageait sur une voie sans retour. Car la République devrait marcher dans le sillage d'un État français, qui allait se révéler une machine de guerre efficace, aux aspirations hégémoniques. L'alliance offensive et défensive telle qu'elle était libellée sur papier impliquait que la République batave devrait entretenir une armée française d'occupation de 25000 hommes, ce qui lui coûterait un million de florins par mois environ. À cela s'ajoutait l'engagement de renforcer l'armée et la flotte en vue de la guerre contre l'Angleterre, ce qui obligeait à créer un poste budgétaire supplémentaire de cinq à dix millions de florins par an.

Tant les conditions de l'alliance française que les dettes héritées de l'Ancien Régime contraignirent les autorités bataves à des dépenses inédites, ce qu'elles ne purent faire qu'en entamant le capital disponible de la République, tout en sachant que cela ébrécherait la fortune des financiers. De nouvelles méthodes de financement s'imposaient afin que soit renflouée la base numéraire nécessaire pour pourvoir au crédit. Aussi les

(2) Voir J. B. MANGER jr., Relations sur les relations économiques entre la France et la Hollande pendant la Révolution française (1785-1795), Paris, 1923, pp. 102-142. Voir aussi, Fr. CROUZET, «Aspects financiers de la relation franco-batave", in A. JOURDAN \& J.Th. LEERSSEN, Remous révolutionnaires. République batave, armée française, Amsterdam, A.U.P., 1996, pp. 49-57.

(3) La République batave avait divisé son «trousseau » en deux parties. La première serait payée essentiellement en traites, tandis que la seconde, d'un montant de 50 millions de florins, serait acquittée en obligations portant intérêt (« rescriptions bataves»). 
Bataves aspiraient-ils à une constitution qui leur permette de combattre effectivement une crise financière qui se prolongeait par trop. Les divergences d'intérêts des provinces, en matière de finances, notamment à l'égard de la fusion des dettes provinciales en une et seule dette nationale, bloquèrent pendant longtemps la réalisation de cette constitution. Il fallut la pression française pour qu'en 1798 fût proclamée la première Constitution unitaire du Peuple batave.

\section{L'époque des impôts nationaux extraordinaires, 1795-1805}

Tant les intérêts de la dette nationale que les dépenses imposées par l'alliance française et la guerre accablèrent le Trésor dans les années 1795-1810 de dépenses qui ne pouvaient être acquittées avec les recettes fiscales habituelles. L'État néerlandais avait à se battre contre un déficit structurel d'une ampleur impressionnante (Tableau 3).

Tableau 3. Vue d'ensemble des dépenses publiques, des intérêts de la dette, des revenus ordinaires et des déficits en millions de florins, 1798-1810. (Pfeil, Ibid., p. 209).

\begin{tabular}{|c|c|c|c|c|}
\hline année & dépenses & intérêts & revenus & déficits \\
\hline 1798 & 63,5 & 36,2 & 25,6 & 38,0 \\
1799 & 84,6 & 29,3 & 33,8 & 50,8 \\
1800 & 78,0 & 34,3 & 33,8 & 44,2 \\
1801 & 72,7 & 32,7 & 33,8 & 38,9 \\
1802 & 66,0 & 32,9 & 33,8 & 32,2 \\
1803 & 73,5 & 33,9 & 35,0 & 38,5 \\
1804 & 72,9 & 35,0 & 35,0 & 37,9 \\
1805 & 72,2 & 33,9 & 35,0 & 37,2 \\
1806 & 82,0 & 34,3 & 42,7 & 39,3 \\
1807 & 78,1 & 34,8 & 46,6 & 31,5 \\
1808 & 80,0 & 38,8 & 44,0 & 36,0 \\
1809 & 70,0 & 38,9 & 48,3 & 21,7 \\
1810 & 74,0 & 41,5 & 42,8 & 31,2 \\
\hline
\end{tabular}

Au cours des ans, cet énorme déficit devait être couvert afin d'éviter le danger imminent d'une banqueroute nationale et de garantir l'existence réelle de la République. Comme l'introduction d'impôts généraux fut freinée dans les années 1795-1805 du fait des divisions partisanes, le nouveau gouvernement fut obligé de souscrire des emprunts (d'anticipation) nationaux ou de lever des impôts extraordinaires. Au début, il chercha à remplir les caisses au moyen d'emprunts volontaires ou de taxations foncières portant intérêt (des emprunts forcés), tandis que les dépenses afférentes (intérêts et remboursement) étaient recouvertes grâce à des prélèvements sur le revenu, ne portant pas intérêt. Tout cela n'était pas de nature à dimi- 
nuer le poids de la dette, ce qui à son tour ne faisait que dévaloriser un peu plus le crédit de l'État (Tableau 4).

Tableau 4. Évolution de la dette néerlandaise et des dépenses en remboursement d'intérêts (en millions de florins) et évolution du quota de déficit (en pourcentage du PNB), 1795-1810. (Pfeil, Ibid., p. 351).

\begin{tabular}{|c|c|c|c|}
\hline année & dette publique & intérêts & quota \\
\hline 1795 & 766 & 22,7 & 160 \\
1800 & $975(+/-)$ & 34,3 & 204 \\
1805 & 1145 & 33,9 & 240 \\
1810 & $1232(+/-)$ & 41,5 & 258 \\
\hline
\end{tabular}

Le gouvernement fut donc malgré tout contraint de faire appel au remède « révolutionnaire », à savoir : une taxe sur la fortune - sans intérêt -, qui entra en application pour la première fois en Hollande en 1801 (4). L'accumulation des impôts sur le revenu et sur la propriété, fixés selon un système proportionnel, signifiait que l'État s'appropriait de plus en plus les ressources privées de la République (tableau 5). Entre 1795 et 1805, les recettes totales des impôts ordinaires (au niveau des provinces) et des impôts extraordinaires (au niveau national) atteignirent ainsi quelque 730 millions de florins.

Tableau 5. La pression des taxes extraordinaires (ou emprunts forcés) dans la période 1788-1810, mesurée en pourcentages selon les revenus et les propriétés de la République. (Pfeil, Ibid.,p.391)

\begin{tabular}{|l|c|l|r|r|c|}
\hline période & $\begin{array}{l}\text { nombre } \\
\text { d'années }\end{array}$ & $\begin{array}{l}\text { recettes } \\
\text { totales }\end{array}$ & $\begin{array}{l}\text { propriété } \\
\text { au total }\end{array}$ & $\begin{array}{l}\text { recettes } \\
\text { moyenne } \\
\text { annuelle }\end{array}$ & $\begin{array}{l}\text { propriété } \\
\text { moyenne } \\
\text { annuelle }\end{array}$ \\
\hline $1788-1794$ & 7 & $0 \%$ & $6 \%$ & $0 \%$ & $0,85 \%$ \\
$1795-1800$ & 6 & $16-21 \%$ & $22,5 \%$ & $2,7-3,5 \%$ & $3,75 \%$ \\
$1801-1805$ & 5 & $34-39 \%$ & $12 \%$ & $6,8-7,8 \%$ & $2,40 \%$ \\
$1806-1810$ & 5 & $0 \%$ & $0,37 \%$ & $0 \%$ & $0,07 \%$ \\
\hline
\end{tabular}

À bien considérer le tableau ci-dessus, on sera stupéfait de l'augmentation inédite des charges imposées aux Bataves à partir de 1795. Si l'on sait qu'un impôt sur la fortune d'un pour cent équivalait à l'époque à une tarification d'impôt sur le revenu d'environ 12,5\%, entre 1795 et 1800, les revenus en principe - étaient annuellement imposés à 49,6\% et 50,4\%, alors que, dans la période précédente, les revenus n'étaient pas taxés au-dessus de 10,6 \%. Si l'on considère les années 1801-1805, le tarif moyen redescend à

(4) Pour un aperçu plus complet des mesures de financement extraordinaires durant la période franco-batave, voir l'annexe B dans T. PFEIL, Tot redding van het vaderland, op. cit., pp. 545-549. 
$37 \%$, d'après nos calculs, mais il faut tout de suite ajouter qu'à partir de 1801 les impôts sur la fortune ne sont plus des emprunts forcés portant intérêt mais des emprunts sans intérêt. Quoi qu'il en soit, jamais dans l'histoire des Pays-Bas, la population n'a été confrontée à une pression fiscale aussi dure.

L'accumulation des impôts extraordinaires fut cause aussi d'une certaine redistribution des rapports en vigueur entre revenus et fortunes. En gros, deux tiers des ménages hollandais échappèrent à ces impositions extraordinaires parce qu'étaient exemptés ceux qui gagnaient moins de 300 florins et ceux qui avaient une fortune inférieure à 500 florins (Tableau 6). Les moins fortunés de la République étaient donc épargnés.

Tableau 6. Pression fiscale moyenne annuelle en florins, par tête (et province) en 1795 et 1805 , subdivisée en revenus au-dessous de $300 \mathrm{fl}$. et en revenus au-dessus de $300 \mathrm{fl}$.

(Pfeil, Ibid., p.393).

\begin{tabular}{|l|c|c|c|c|}
\hline & $\begin{array}{l}\text { pression moyenne } \\
\text { par tête, en 1795 }\end{array}$ & $\begin{array}{l}\text { pression moyenne } \\
\text { par tête en 1805 } \\
\text { (revenus < 300 fl.) }\end{array}$ & $\begin{array}{l}\text { pression moyenne } \\
\text { par tête en 1805 } \\
\text { (revenus > 300 fl.) }\end{array}$ & $\begin{array}{l}\text { différence } \\
\text { selon revenus } \\
\text { en 1805 }\end{array}$ \\
\hline Hollande & 23,87 & 27,92 & 99,90 & 71,98 \\
Gelderland & 8,17 & 8,49 & 28,19 & 19,70 \\
Zélande & 19,28 & 20,76 & 60,56 & 39,80 \\
Utrecht & 19,54 & 22,18 & 82,63 & 60,45 \\
Frise & 19,80 & 16,98 & 53,29 & 36,31 \\
Groningue & 13,21 & $\mathbf{1 1 , 6 2}$ & 36,22 & 24,60 \\
Overijssel & 7,91 & 8,01 & 27,96 & 19,95 \\
Drenthe & 6,07 & 6,28 & 26,23 & 19,95 \\
Brabant & 5,83 & 5,91 & 14,99 & 9,08 \\
Total & $\mathbf{1 6 , 9 1}$ & $\mathbf{1 8 , 1 3}$ & $\mathbf{6 1 , 6 6}$ & $\mathbf{4 3 , 5 3}$ \\
\hline
\end{tabular}

Les données du tableau 6 montrent que la pression fiscale moyenne par tête d'habitant, dans les foyers ayant des revenus inférieurs à 300 florins, et, à l'exception des habitants des provinces de Groningue et de Frise, augmente légèrement entre 1795 et 1805, tandis que la pression moyenne sur les revenus au-dessus de 300 florins se multiplie quasiment par quatre. De même sautent aux yeux les écarts géographiques. La part de la Hollande dans le produit des impôts extraordinaires s'élève fort au-dessus de sa part dans le produit moyen des impôts ordinaires. Pour cela, il y a au moins deux explications. Tout d'abord, le salaire moyen était plus élevé en Hollande que dans les autres provinces, ce qui fait qu'il y avait proportionnellement moins de contribuables qui jouissaient du régime d'exemption. Deuxièmement, et d'après les observateurs contemporains, il n'était pas rare que les régions peu peuplées se soustraient aux levées fiscales successives. Le produit des impôts y demeurait fort en deça des prévisions. Cela vaut notamment pour les provinces de Groningue, Frise, Drenthe, Overijssel, Gelderland et Brabant. 


\section{L'époque de l'uniformisation des impôts, 1806-1810}

Après la reprise des hostilités en Europe (1803), la République batave était au bord de la banqueroute. D'une part, le rendement des impôts extraordinaires déclinait, d'autre part, les dépenses militaires explosaient. Les tentatives pour réduire les déficits se heurtaient à la volonté de Napoléon pour que soient au contraire intensifiés les efforts militaires bataves. Sur son ordre, le gouvernement néerlandais fut invité par le traité du 25 juin 1803 à reconfirmer son alliance avec la France, à s'engager à entretenir une armée française de 18000 hommes et à constituer une armée batave de 16000 soldats. La marine eut à pâtir d'exigences plus brutales encore. Avant 1804, elle devait fournir 5 vaisseaux de ligne, 5 frégates équipées, 100 petites chaloupes canonnières et 250 barges propres au transport de troupes.

Dans ce contexte, il ne restait plus au gouvernement hollandais qu'à se tourner une fois de plus vers les fondements du système fiscal batave. Si les recettes fiscales ordinaires n'étaient pas augmentées considérablement, les dépenses nationales risquaient d'être une fois de plus dépendantes des impôts extraordinaires sur le revenu et sur la fortune, qui non seulement étaient extrêmement impopulaires, mais pesaient également et de façon disproportionnée sur les classes moyennes actives.

Avec l'introduction en 1805 d'une constitution centralisée et la nomination à la tête de la République d'un Grand Pensionnaire, Rutger Jan Schimmelpenninck, avait sonné l'heure d'une réforme de fond de la fiscalité batave. Bien que le nouveau régime n'ait pas proclamé impérieusement que devaient être prioritaires l'introduction d'un système fiscal uniforme et la réorganisation de l'administration intérieure, les cercles officiels ne doutaient plus de leur nécessité. De ce point de vue, le retour au gouvernement de l'unitariste Isaac Gogel, qui, en tant qu'agent des Finances, avait dès 1799 présenté à l'Assemblée nationale batave un projet de réforme fiscale unitaire, disait bien de quel côté allait pencher la balance. Pour Gogel, seul un système fiscal uniforme pouvait sauver la République et lui rendre son rang parmi les puissances européennes.

Le système conçu par Gogel fut mis en place en 1806. Avec lui furent uniformisés en Hollande les impôts et les tarifs (5). La centralisation fiscale et la réorganisation de l'administration intérieure nécessaire pour que fonctionne le nouveau système mirent fin au pouvoir politique qu'avaient encore conservé les autorités provinciales dans l'administration de l'État unitaire. L'introduction du «Système général» paracheva l'unification politique et

(5) Les éléments les plus importants du système étaient un impôt sur les biens immobiliers, un impôt personnel sur les diverses sources de richesses, quelques droits sur les timbres et quelques impôts indirects. 
financière de l'ancienne confédération des Provinces-Unies, amorcée en 1795 avec la proclamation de la République batave.

Grâce aux revenus que devait fournir le système général, le gouvernement pensait augmenter ses moyens d'action, puisqu'il pourrait désormais payer les dépenses ordinaires avec les recettes fiscales ordinaires. De ce point de vue, le système ne répondit pas tout à fait aux attentes. Alors qu'il était prévu que rentrent 49 millions de florins, les recettes ne s'élevèrent en 1806 qu'à 42 millions et en 1807 à 44,6 millions. Il n'en demeure pas moins qu'en 1807 le nouveau système avait rapporté 9,6 millions de plus que l'ancien, ce qui impliquait une augmentation de $27 \%$ et ce qui constituait malgré tout un progrès, étant donné le malaise économique qui sévissait dans le pays (Tableau 7).

Tableau 7. Recettes prévues et réalisées des impôts généraux et des droits de douanes (convois et licences) en 1806 et 1807 (en millions de florins). (Pfeil, Ibid., p. 466.)

\begin{tabular}{|l|c|c|c|}
\hline & prévisions 1806 & recettes 1806 & recettes 1807 \\
\hline immobilier & 11,0 & 9,5 & 9,2 \\
mobilier & 5,0 & 4,7 & 4,4 \\
succession & 3,2 & 1,2 & 2,1 \\
divers (6) & 2,7 & 2,8 & 3,0 \\
indirects (7) & 17,7 & 16,7 & 17,1 \\
timbres & 6,0 & 5,0 & 5,7 \\
douanes & 3,5 & 2,8 & 3,0 \\
Total & $\mathbf{4 9 , 1}$ & $\mathbf{4 2 , 7}$ & $\mathbf{4 4 , 6}$ \\
\hline
\end{tabular}

Les gains réalisés dans les recettes fiscales proviennent surtout des taxes personnelles, des taxes indirectes sur le vin et les alcools et des nouveaux droits sur les timbres. Le bénéfice fiscal signifiait également une pression accrue sur les citoyens (8). C'est ainsi que le produit fiscal moyen passa de 16,90 florins en 1795 à 19,59 florins par tête d'habitant. De même, les recettes des impôts indirects s'accrurent considérablement, même si, en proportion, la part des impôts directs augmenta plus encore dans les recettes réalisées par le nouveau système. Mais cela ne remédia toujours pas aux écarts entre les provinces (Tableau 8).

(6) Cette rubrique comprend le mobilier, le bétail, les dépenses somptuaires, les traitements des domestiques. En 1807, s'y ajoute une taxe sur les cheminées.

(7) Parmi les impôts indirects se trouvent le charbon, la tourbe, la mouture, le sel, l'alcool, le vin, le bétail, le savon, le tonnage et le poids public.

(8) N'oublions pas de mentionner que les impôts extraordinaires avaient été supprimés lors de la mise en œuvre du nouveau système fiscal. 
Tableau 8. Recettes fiscales par tête d'habitant dans les provinces (1795 et 1807, en florins). (Pfeil, Ibid, p. 467)

\begin{tabular}{|l|c|c|c|}
\hline & $\begin{array}{c}\text { recettes par tête } \\
\mathbf{1 7 9 5}\end{array}$ & $\begin{array}{c}\text { recettes par tête } \\
\mathbf{1 8 0 7}\end{array}$ & $\begin{array}{c}\text { indice de recettes } \\
\mathbf{1 8 0 7}(\mathbf{1 7 9 5}=\mathbf{1 0 0})\end{array}$ \\
\hline Hollande & 22,84 & 29,78 & 130 \\
Gelderland & 7,14 & 8,15 & 114 \\
Overijssel & 6,88 & 9,29 & 135 \\
Groningue & 12,18 & 14,94 & 123 \\
Zélande & 18,25 & 19,92 & 109 \\
Frise & 18,77 & 23,31 & 124 \\
Utrecht & 18,51 & 26,68 & 144 \\
Drenthe & 5,4 & 8,97 & 178 \\
Brabant & 4,80 & 7,89 & 164 \\
Total & $\mathbf{1 6 , 9 0}$ & $\mathbf{1 9 , 5 9}$ & $\mathbf{1 1 6}$ \\
\hline
\end{tabular}

Parmi les provinces qui étaient autrefois le plus faiblement pressurées par la fiscalité, c'est au Brabant et en Drenthe que le produit de l'impôt (par tête) augmenta le plus. Surprenant est aussi le fait que les gains fiscaux réalisés dans la province de Gelderland soient fort inférieurs à ceux de la Hollande, la province ayant depuis toujours été le plus imposée. En fin de compte, le nouveau système fiscal ne détruisit pas du tout au tout les anciennes inégalités entre les provinces. La part de la Hollande dans le produit fiscal total passa de 59,7 à $57,4 \%$, ce qui ne fait pas une grande différence. À l'inverse, la part moyenne des provinces de terre augmenta de $3,27 \%$ à $3,75 \%$.

\section{La banqueroute nationale de 1810}

Aucune décision gouvernementale n'a contribué plus à la modernisation de l'État néerlandais, financier et fiscal que l'introduction en 1805 d'une fiscalité uniforme. Pourtant, le produit du système général était loin de suffire à combler les déficits créés par les dépenses (militaires) annuelles. Aucune mesure financière ne semblait pouvoir apporter des recettes supplémentaires. Il revint à Louis Bonaparte, roi de Hollande depuis juin 1806, de chercher une issue à cette impasse financière (9). Le problème était que l'alliance militaire avec la France et la demande incessante de crédits militaires rendaient impossible toute économie sur les dépenses de l'armée, de la

(9) Le régime fort bref du Grand Pensionnaire fut donc le dernier gouvernement de la République batave, qui fut remplacée en 1806 par le Royaume de Hollande. 
flotte et de la dette publique. La politique néerlandaise n'avait, quoi qu'il en soit, aucune marge de manœuvre, puisque, par sa position géographique, la Hollande était d'une importance stratégique pour le gouvernement français, dans sa lutte contre l'Angleterre. Le roi Louis lui-même n'y put rien changer. Tous ses efforts pour réduire les dépenses de guerre furent anéantis sans pardon par Napoléon. Parvenir à équilibrer les dépenses nationales était donc une entreprise vouée à l'échec, d'autant que lorsque vit le jour la quatrième coalition de 1806, les dépenses de guerre culminèrent une fois de plus. La poursuite de la guerre en Europe condamna le régime de Louis à être sans cesse accablé par le manque d'argent, ce qui le contraignit à chercher des solutions coûteuses ou impopulaires, afin d'éviter la banqueroute. Chaque année, le Roi se voyait obligé d'ordonner des paiements forcés par avance ou par anticipation sur les emprunts projetés ou impôts à venir, dont la taxe immobilière. Parallèlement, on n'hésitait pas à hypothéquer plusieurs fois de suite des effets non encaissés ou des récépissés accordés provisoirement lors des levées d'argent. De telles pratiques n'étaient pas le fait des autorités elles-mêmes mais de banquiers renommés.

Ces expédients ne suffisaient pas à assurer au Trésor les fonds nécessaires. Sans cesse, le Roi dut remettre à plus tard le paiement des intérêts. Aussi, lors de la dernière année de son existence, le Royaume de Hollande devait trente millions de florins à ses créanciers. En 1810, il dut mettre en circulation six millions de florins en papier-monnaie (quittances d'impôts immobiliers) - qui seraient acceptés ou échangés contre de l'argent comptant, lorsque seraient payés les impôts immobiliers de l'année 1810 - afin de pouvoir remplir quelques-unes de ses obligations. Par ailleurs, à partir d'avril 1808, le Roi fut contraint de payer la plupart des dépenses nationales (intérêts, traitements, rentes) pour moitié avec des récépissés et du papiermonnaie. Ces signes annonçaient une banqueroute nationale. En mars 1808, Louis avait lancé un emprunt forcé de trente millions de florins à $7 \%$ d'intérêt, dont les frais annuels seraient répartis sur les divers départements, à raison d'un quota de trois millions de florins. Il rapporta seulement vingt millions de florins tandis que le cours des obligations tomba en chute libre malgré tous les soutiens officiels. Pour obtenir les dix millions de florins qui lui manquaient, d'une part, et pour couvrir le déficit prévu pour les années 1808-1809, d'autre part, le gouvernement essaya de lancer un emprunt volontaire de vingt millions de florins, qui n'eut pas plus de succès. La monarchie hollandaise ne pouvait plus alors éviter la banqueroute et l'invalidation d'une partie de ses créances. Pour le marché des capitaux, tout était perdu. Les cours des obligations en souffrance chutèrent à la fin de l'année 1809 au-dessous du seuil magique de $30 \%$.

En 1810, le rideau tomba sur la politique réformatrice batave, qui visait essentiellement à assurer à la République ou au royaume son autonomie et à éviter la banqueroute. Ces idéaux nationaux durent céder le pas devant le 
joug napoléonien, qui avait transformé la Hollande en une caisse d'escompte, apte à renflouer la machine de guerre impériale. En juillet 1810, le Royaume fut annexé à la Grande Nation. Aussitôt Napoléon y appliqua un remède de cheval : la «tiërcering», soit la répudiation des deux tiers de la dette, pour que soit ramené à des proportions raisonnables le gigantesque, déficit de 42 millions de florins. De ce fait, il réalisa une banqueroute partielle, à laquelle avaient répugné pour des raisons diverses les politiques néerlandais.

(Traduction du néerlandais : A. Jourdan) 